\title{
Endoscopic Management of Penetrated Adjustable Gastric Band with Its Connecting Tube: Report of a Case
}

\author{
Halil Coskun ${ }^{a}$ Suleyman Bozkurt ${ }^{a}$ Tuba Atak $^{b}$ Huseyin Kadioglu ${ }^{a}$ \\ ${ }^{a}$ Department of Surgery, Faculty of Medicine, Bezmi Alem Vakif University, \\ ${ }^{\mathrm{b}}$ Department of Surgery, SB Istanbul Göztepe Training and Research Hospital, Istanbul, Turkey
}

\author{
Keywords \\ Gastric banding · Complication - Migration . \\ Band-cutter · Endoscopy
}

\section{Summary}

Aim: Laparoscopic adjustable gastric banding (LAGB) is a widely performed surgical procedure for the treatment of morbid obesity. Late complications mainly originate from either the injection port or the gastric band. Complications from the tube and band together are also described. Case Report: We here report a case of adjustable gastric band migrated subserosally into the stomach with its whole connecting tube, which was removed endoscopically using a band cutter. Conclusion: In selected patients endoscopic techniques in combination with minimally invasive procedures can safely be used in the treatment of complete gastric band migration.

\section{Introduction}

Obesity is currently considered as a state of disease, because of its medical, physical, social, economic and psychological co-morbid conditions [1]. Laparoscopic adjustable gastric banding (LAGB) is the most common bariatric procedure performed for the treatment of morbid obesity. Complications of LAGB are rare and might be related to the reservoir, the connecting tube and the band itself. Band erosions and migrations are one of them. The management of such late complications are usually surgical interventions, but sometimes the endoscopic route is attempted [2]. We here report the management of a penetrated band with its loose connecting tube into the stomach by means of a band cutter 2 years after removal of an infected injection port.

\section{Case Report}

A 35-year-old obese man (weight $125 \mathrm{~kg}$, BMI $42.6 \mathrm{~kg} / \mathrm{m}^{2}$ ) underwent LAGB (MIDBAND ${ }^{\otimes}$, Médical Innovation Développement, Dardilly, France) 4 years ago in another medical center. The postinterventional follow-up showed considerable weight loss; within in 2 years the patient reduced his BMI to $28.9 \mathrm{~kg} / \mathrm{m}^{2}$. But the injection port was explanted because of reservoir infection leaving the gastric band and tubing intraabdominal. For the next 2 years the patient gained weight and was admitted to our center for checking the activity of gastric band with a BMI $37.7 \mathrm{~kg} / \mathrm{m}^{2}$. Upper gastrointestinal system endoscopy was performed which revealed a nearly complete penetration of the gastric band with its connecting tube into the gastric wall (fig. 1). Abdominal CT scan confirmed that the gastric band was located subserosally in the stomach wall (fig. 2). Because the band is almost completely penetrated, an endoscopic approach was felt to be safe and feasible.

\section{Procedure}

The intervention was performed under sedation anesthesia. Under endoscopic view the tissue bridge over the band was cut intragastrically step by step using a endoscopic needle knife papillotome. Thereafter, the endoscopically applied cutting wire was placed between the band and gastric wall. The free ends of the cutting wire were taken out, and a loop of wire was created around the band. The cutting wire was inserted into the flexible wire guide which in the beginning is only loosely tightened. Then the toggle was applied, and with small tours of the handle the band was cut. The tube and the connecting tube slipped into the gastric lumen. The band was cut using an AMI gastric banding cutter (C.J.Medical, Haddenham, UK) and finally retrieved endoscopically by a polypectomy snare (fig. 3). No subdiaphragmatic free air was detected on plain abdominal $\mathrm{X}$-ray (fig. 4). The postinterventional course was uneventful; the patient was discharged on the same day.

\section{KARGER}

Fax +497614520714

Information@Karger.de

www.karger.com
(C) 2011 S. Karger GmbH, Freiburg

$1662-4025 / 11 / 0043-0244 \$ 38.00 / 0$

Accessible online at:

www.karger.com/ofa
Suleyman Bozkurt MD

Tepeören M. Istanbulpark

C. Arkeon Evleri Sit. No:8 H67 Akfirat Tuzla 34959 Istanbul, Turkey

Tel. +90 5323661686, Fax +90 2165660294

suleymanbozkurt65@gmail.com 


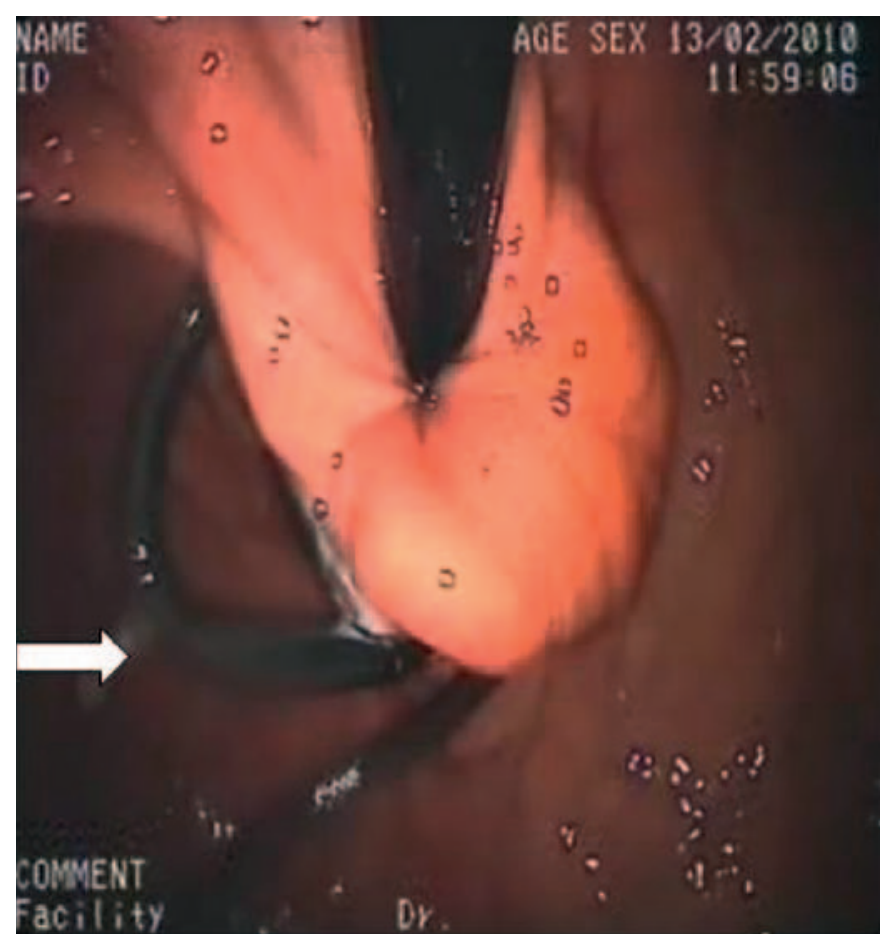

Fig. 1. Endoscopic view of gastric band and its connecting tube in the gastric lumen.

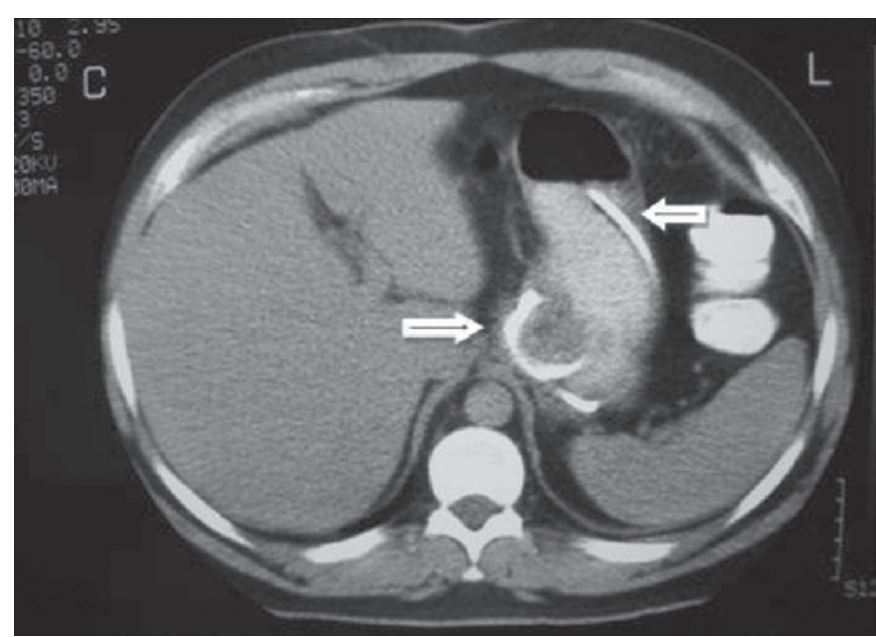

Fig. 2. CT scan showing the subserosal gastric band and intragastric connecting tube.

\section{Discussion}

LAGB has become the most popular bariatric procedure in the world during the past decade because the laparoscopic procedure is relatively simple without visceral transection or anastomosis, the band can easily be adjusted, the whole procedure is almost completely reversible, and patients could be discharged early from the hospital. On the other hand there are also some disadvantages of gastric banding, introduction of foreign material into the abdomen being one of these [3]. In a huge number of publications, gastric banding has been demonstrated to have

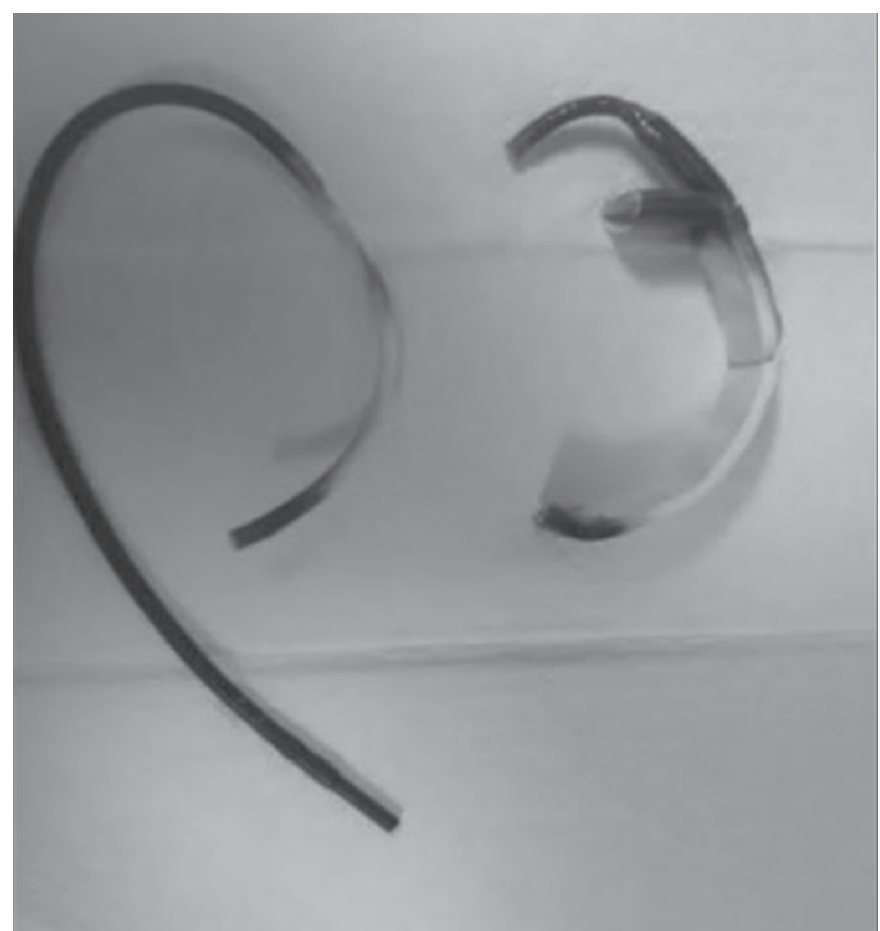

Fig. 3. View of the endoscopically explanted gastric band and its connecting tube.

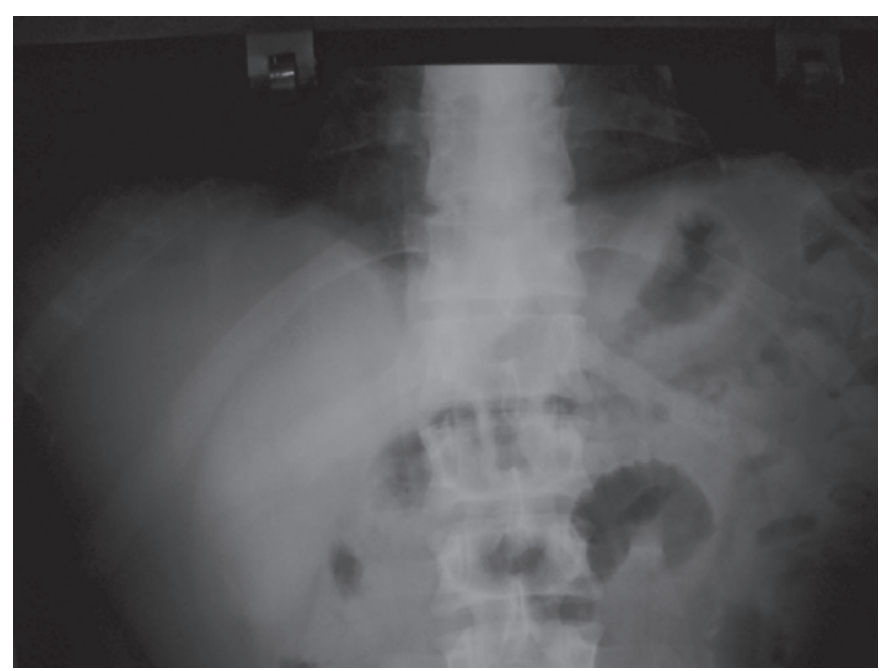

Fig. 4. Plain abdominal $X$-ray after the procedure.

a significant incidence of complications including acute slippage of the gastric wall, irreversible pouch dilation, esophageal dilation, band erosion, subcutaneous port infection, leak, disconnection of the port-catheter system, and port twist. The incidence of these complications has been reported to be $6-26 \%$ and has led to a mean re-operation rate of 2-40\% [4-6]. Late complications mainly originate from either the injection port (dislocation, infection, leakage) or the gastric band (pouch dilatation, slippage, leakage, gastric erosion). 
Band migration through the gastric wall is a rare and serious complication of LAGB (0.3-11\%) [7-10] and can occur due to early or late erosion of the gastric wall [11]. Early erosions can be caused by a damage to the gastric wall during initial operation and are always associated with serious infections. Late erosions are the result of a destructive process followed by an effective self-healing of the gastric wall; they are usually associated with no or very small infections [12]. Pressure applied to the gastric wall is regarded as the primary etiologic factor for band penetration. External pressure is applied either through chronic overfilling of the banded stomach or by inclusion of too much gastric wall during the operation. Internal pressure is applied as a result of ingestion of excessively large food boluses early after operation. A rejection reaction against the silicon gastric band with subsequent circumferential fibrous contraction is also discussed [2]. Third, the surface of the band used and its diameter could may also play a role the development of band migration/erosion [9].

Gastric band erosion mostly occurs within 12 months. However, because the clinical symptoms are generally mild and transient, they frequently remain undiagnosed [8, 10, 13]. Especially in case of a port site infection gastric band erosion must be ruled out. Unexplained weight gain, epigastric pain, and port infection most frequently accompany band erosion $[8,9]$. In our case it would have been advisable to check the position and function of the band when the infected port was extracted, but according to the patient no attempt was made to also test the functionality of the band.

There is a multitude of reports on band migration varying from a visible gastric band at endoscopy to total band migration. Nocca et al. [8] classified the degree of migration into three stages:

- stage I: a small part of the band visible through a hole in the gastric mucosa

- stage II: partial migration (more than half of the band free in the gastric lumen

- stage III: complete migration (band and tube).

Removal of the band is mandatory and can be done by surgery or endoscopy. Endoscopic removal is feasible provided that band has partially or totally migrated. Nevertheless, it requires specific equipment and a skilled endoscopist [8].

Any serious late complication at the port site may end up with the surgical removal of the port leaving the deflated band and tubing intraabdominally. If the tube was totally migrated into the gastric lumen, complications such as strangulation around mesenteric root or obstruction of the jejunal loops or migration to the colon or to the hilus of the kidney have been described [14-16].

The diagnosis of a penetrated gastric band is usually established by gastroscopy or X-ray control. CT scans are the most important diagnostic cornerstone in determining the position of the band and its connecting tube in the stomach wall. Barium contrast radiography provides additional information on other complications of gastric banding such as band slippage or pouch dilatation [17].

In almost all cases, a penetrated band was removed surgically; to our knowledge, there are only few case reports of endoscopic management of a penetrated gastric band $[2,10]$. Whether or not a penetrated gastric band can be managed endoscopically depends on the degree of penetration. The risk of gastric perforation after endoscopic management is higher in patients with small or partial penetration of the band. Thus, the optimal candidate for endoscopic therapy should have an almost complete penetration of the band. In this group of patients, the risk of an iatrogenic perforation is relatively small. As demonstrated by the present case, endoscopic management of a penetrated gastric band is feasible under certain circumstances. According to some authors [11, 12], complete band migration can be awaited allowing for endoscopic retrieval because, compared with operative removal, the procedure described is expected to have fewer complications. Of course this approach is acceptable in an asymptomatic and informed patient only.

In this report, we describe the successful endoscopic removal of a gastric band and its connecting tube which were migrated into the stomach, demonstrating that endoscopic techniques in combination with minimally invasive procedures can safely be used in the treatment of complete gastric band migration. Even if gastric band removal is a routine intervention, it is advisable to perform it in a hospital because of possible complications.

\section{Disclosure Statement}

The authors declare no conflict of interest.

\section{References}

1 Stroh C, Hohmann U, Will U, Flade-Kuthe R, Herbig B, Höhne S, et al: Experiences of two centers of bariatric surgery in the treatment of intragastral band migration after gastric banding - the importance of the German multicenter observational study for quality assurance in obesity surgery 2005 and 2006. Int J Colorectal Dis 2008;23:901-908.
De Palma GD, Formato A, Pilone V, Rega M, Giuliano ME, Simeoli I, et al: Endoscopic management of intragastric penetrated adjustable gastric band for morbid obesity. World J Gastroenterol 2006; 12:4098-4100.

>3 Biertho L, Steffen R, Ricklin T, Horber FF, Pomp A, Inabnet WB, et al: Laparoscopic gastric bypass versus laparoscopic adjustable gastric banding: a comparative study of 1,200 cases. J Am Coll Surg 2003;4:536-547.
4 Chevallier JM, Zinzindohoue F, Douard R, Blanche JP, Berta JL, Altman JJ, et al: Complications after laparoscopic adjustable gastric banding for morbid obesity: experience with 1,000 patients over 7 years. Obes Surg 2004;14:407-414.

5 Buchwald H, Oien DM: Metabolic/bariatric surgery worldwide 2008. Obes Surg 2009;19:1605-1611 
6 Fried M, Miller K, Kormanova K: Literature review of comparative studies of complications with Swedish Band and Lap-Band ${ }^{\circledR}$. Obes Surg 2004;14:256-260.

7 Niville E, Dams A, Vlasselaers J: Lap-Band erosion: incidence and treatment. Obes Surg 2001;11:744-747.

8 Nocca D, Frering V, Gallix B, de Seguin des Hons C, Noel P, Pierredon Foulonge MA, et al: Migration of adjustable gastric banding from a cohort study of 4,236 patients. Surg Endosc 2005;19:947950.

9 Naef M, Mouton WG, Naef U, Kummer O, Muggli B, Wagner HE: Graft survival and complications after laparoscopic gastric banding for morbid obesity - Lessons learned from a 12 year experience. Obes Surg 2010; DOI: 10.1007/s11695-010-0205-0.
10 Lattuda E, Zappa MA, Mozzi E, Fichera G, Granelli P, De Ruberto F, et al: Band erosion following gastric banding: How to treat it. Obes Surg 2007:17:329-333.

11 Zehetner J, Holzinger F, Triaca H, Klaiber C: A 6-year experience with the Swedish adjustable gastric band prospective long-term audit of laparoscopic gastric banding. Surg Endosc 2005;19:21-28. 12 Abu-Abeid S, Bar Zohar D, Sagie B, Klausner J: Treatment of intra-gastric band migration following laparoscopic banding: safety and feasibility of simultaneous laparoscopic band removal and replacement. Obes Surg 2005;15:849-852.

3 Angus LDG, Rizvon K, Zhou D, Seitelman E, Cardoza S: Intra-gastric band erosion from an uninflated Lap-Band: a case report. Obes Surg 2008; 18:1636-1639.
14 Tekin A: Migration of the connecting tube into small bowel after adjustable gastric banding. Obes Surg 2010;20:526-529.

15 Bell BJ, Myers KM, Bour ES: Intracolonic tubing migration: an unusual complication of the silastic adjustable gastric band (LapBand). Surg Obes Relat Dis 2007;3:486-487.

16 Sneijder R, Cense HA, Hunfeld M, Breederveld RS: A rare complication after laparoscopic gastric banding: Connecting-tube penetration into the hilus of the kidney. Obes Surg 2009;19:531-533.

17 Hainaux B, Agneessens E, Rubesova E, Muls V Gaudissart Q, Moschopoulos C, et al: Intragastric band erosion after laparoscopic adjustable gastric banding for morbid obesity: Imaging characteristics of an underreported complication. AJR Am J Roentgenol 2005;184:109-112. 